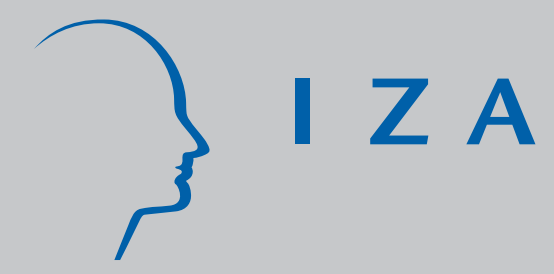

IZA DP No. 4125

Negative Externalities and Equilibrium Existence in Competitive Markets with Adverse Selection

Ferdinand von Siemens

Michael Kosfeld

April 2009 


\title{
Negative Externalities and Equilibrium Existence in Competitive Markets with Adverse Selection
}

\author{
Ferdinand von Siemens \\ University of Amsterdam \\ Michael Kosfeld \\ University of Frankfurt \\ and IZA
}

Discussion Paper No. 4125

April 2009

IZA

P.O. Box 7240

53072 Bonn

Germany

Phone: +49-228-3894-0

Fax: +49-228-3894-180

E-mail: iza@iza.org

\begin{abstract}
Any opinions expressed here are those of the author(s) and not those of IZA. Research published in this series may include views on policy, but the institute itself takes no institutional policy positions.

The Institute for the Study of Labor (IZA) in Bonn is a local and virtual international research center and a place of communication between science, politics and business. IZA is an independent nonprofit organization supported by Deutsche Post Foundation. The center is associated with the University of Bonn and offers a stimulating research environment through its international network, workshops and conferences, data service, project support, research visits and doctoral program. IZA engages in (i) original and internationally competitive research in all fields of labor economics, (ii) development of policy concepts, and (iii) dissemination of research results and concepts to the interested public.
\end{abstract}

IZA Discussion Papers often represent preliminary work and are circulated to encourage discussion. Citation of such a paper should account for its provisional character. A revised version may be available directly from the author. 
IZA Discussion Paper No. 4125

April 2009

\section{ABSTRACT}

\section{Negative Externalities and Equilibrium Existence in Competitive Markets with Adverse Selection*}

Rothschild and Stiglitz (1976) show that there need not exist a competitive equilibrium in markets with adverse selection. Building on their framework we demonstrate that externalities between agents - an agent's utility upon accepting a contract depends on the average type attracted by the respective principal - can solve the equilibrium existence problem, even when the size of the externalities is arbitrarily small. Our result highlights the degree of control a principal has over the attractiveness of his contracts as an important feature for equilibrium existence, thereby offering a new perspective on existing theories of competition in markets with adverse selection.

JEL Classification: D82, D86

Keywords: asymmetric information, competition, adverse selection, externality

Corresponding author:

Michael Kosfeld

Department of Economics and Business Administration

Johann Wolfgang Goethe-University Frankfurt

Dantestr. 9

D-60325 Frankfurt am Main

Germany

E-mail: kosfeld@econ.uni-frankfurt.de

Financial support by the University of Zurich, the Swiss State Secretariat for Education and Research, and the German Science Foundation (DFG) through the SFB/TR 15 is gratefully acknowledged. 


\section{Introduction}

In their seminal contribution to the economics of imperfect information, Rothschild and Stiglitz (1976) show that there need not exist a competitive equilibrium in markets with adverse selection. We introduce a simple externality between agents into their framework and show that this externality can solve the equilibrium existence problem. In our model, an agent's utility upon accepting a contract does not only depend on his type and the contract, but also on the average type of agents attracted by the respective principal. We prove that such externality can ensure the existence of equilibrium even if the externality is arbitrarily small. Further, any competitive equilibrium is a Pareto-dominant separating equilibrium. Although externalities facilitate equilibrium existence, they thus do not blunt model predictions.

To illustrate, consider a screening version of Spence's (1973) labor market model. Suppose firms compete for workers by offering employment contracts. Employment contracts specify education levels which make an employee eligible for particular wages. Workers have private information on their productivity. As high-productivity workers have lower costs of acquiring education than low-productivity workers, firms can separate workers by appropriately combining education levels and wages. Rothschild and Stiglitz (1976) show that in a competitive equilibrium all firms make zero profits, low-productivity workers acquire no education, and high-productivity workers acquire the minimum education needed to ensure separation from low-productivity workers. The resulting screening costs do not depend on the fraction of low-productivity workers, and are borne by the high-productivity workers. Now suppose a firm enters the market with a pooling contract that requires no education and sets wages so as to make some small profit when accepted by all workers. This contract becomes more and more attractive as the fraction of low-productivity workers decreases, and it converges to the best possible contract that can be offered to high-productivity workers without making losses as the fraction of low-productivity workers and the offering firm's profits go to zero. If the fraction of low-productivity workers is sufficiently small, such a pooling contract thus attracts all workers and makes positive profits. There then exists no competitive equilibrium.

Now suppose that there are externalities. To makes things concrete, suppose that after a worker has joined some firm he is matched into a team with another employee of the same firm. The two workers are promised a bonus if the team succeeds to meet a performance target. Like in Kremer (1993) production consists of many tasks, all of which must be well ex- 
ecuted for the team to be successful. ${ }^{1}$ High-productivity workers can do the job, whereas the presence of at least one low-productivity worker causes the team to fail. High-productivity workers then dislike being matched with a low-productivity colleague. Low-productivity workers, on the other hand, do not care for their colleague's productivity as their own presence is already sufficient to ensure that they never get the team bonus. A high-productivity worker's utility upon accepting the offered employment contract thus depends on the fraction of low-productivity workers employed by the same firm. For low-productivity workers this is not the case.

The externality between agents entails that there exist equilibrium acceptance choices in which a firm offering a pooling contract is not able to attract any high-productivity workers. Given that none of the other high-productivity workers accepts the contract, each highproductivity worker finds it optimal not to accept the contract as he would be matched with a low-productivity colleague for sure, which he dislikes. As the pooling contract makes losses when attracting only low-productivity workers, market entry is unprofitable. Hence, a competitive equilibrium exists. Our main result shows that the externality does not have to be large in order to ensure equilibrium existence. In fact, it can become arbitrarily small. The reason is that firms can offer what we call "preemptive contracts." These contracts, which in equilibrium will be offered by firms drawing only low-productivity workers, are not accepted by any workers in equilibrium. However, following the market entry of a new firm attracting only low-productivity workers, they suddenly become appealing to high-productivity workers. We show that the maximum utility a high-productivity worker can get by accepting a preemptive contract after market entry can be unbounded even if the externality is arbitrarily small. Therefore, equilibrium existence is guaranteed.

Our analysis offers a new perspective on existing theories of competition in markets with adverse selection. Extending Rothschild and Stiglitz's original equilibrium concept Wilson (1977) and Riley (1979) allow principals to react to market entry. While this solves the equilibrium existence problem, equilibrium characteristics depend on whether principals can add or withdraw contracts after market entry. As argued by Hellwig (1987) explicitly modeling

\footnotetext{
${ }^{1}$ Kremer (1993) motivates such strongly complementary production functions by referring to the "space shuttle Challenger which had thousands of components: it exploded because it was launched at a temperature that caused one of those components, the O-rings, to malfunction." (p. 551) Further examples of O-ring production functions are discussed in Dalmazzo (2002), Fabel (2004), and Jones (2008).
} 
the principals' strategic interaction generates interesting insights and solves the equilibrium existence problem; however, equilibrium predictions remain "very sensitive to the details of the game-theoretic specification" (p.320). ${ }^{2}$ Gale (1992) and Dubey and Geanakoplos (2002) depart from what the latter criticize as the "hybrid oligopolistic-competitive story" and take a Walrasian approach. In the Walrasian approach, market participants do not act strategically but consider the other parties' behavior as unaffected by their own actions. Contracts are traded like consumption commodities, and the attractiveness - so to speak the price - of a contract is determined by the forces of supply and demand rather than by the decision of principals. These assumptions guarantee equilibrium existence. Adverse selection arises in such a setting if the attractiveness of a contract depends on the types of the market participants who accept this contract.

In a sense, the externality we study in our paper is of exactly the same kind as in the Walrasian approach. However, while in a Walrasian market the externality is inextricably connected to the existence of adverse selection, we can vary the strength of the externality keeping the original adverse selection problem fixed. This allows us to isolate the impact of the externality on equilibrium existence from the effect of changing the equilibrium concept. In fact, we derive sufficient and necessary conditions on the externality ensuring equilibrium existence in the original Rothschild and Stiglitz (1976) setup. Our study thereby highlights a central feature of the Walrasian approach being of key importance for the existence of equilibrium: the degree of control a principal has over the attractiveness of his offered contracts. ${ }^{3}$ In a Rothschild-Stiglitz setup, an agent's utility upon accepting a contract depends exclusively on the contract terms and the agent's type. This gives a principal full control over the attractiveness of a contract for a particular type of agent. Market entry is possible as principals can make sure that all types of agents find it optimal to accept the new pooling contract. In contrast, in a Walrasian setup demand and supply rather than principals determine a contract's attractiveness. This restrains market entry and facilitates equilibrium existence. In our model, principals can at least partly commit to and thus control certain contract terms, i.e., a contract's attractiveness is not primarily determined by market forces. However, due to the externality one important aspect affecting the attractiveness of a contract lies beyond a principal's control: the distribution of agents across contracts. Similar to the Walrasian model this lack of control ensures equilibrium existence. In contrast to the Walrasian model

\footnotetext{
${ }^{2}$ See also Bester (1985) and Cho and Kreps (1987).

${ }^{3}$ See also the discussion in Dubey and Geanakoplos (2002), pp. 1549-1550.
} 
lack of control in our model can become arbitrarily small without eliminating the adverse selection problem.

\section{Model}

\subsection{Principals, Agents, and Contracts}

There is a countably infinite number of principals facing a continuum of agents with total mass normalized to one. Let $n \in \mathbb{N}$ be the identity of a principal. Let $\Theta=\{\ell, h\} \in \mathbb{R}^{2}$ be the agents' type space with $h>\ell$. Types are private information and independent, where initially each agent is of type $h$ with probability $\left.\mu_{0} \in\right] 0,1[$.

Principals can enter the market at zero costs by offering the agents countable - possibly infinite - sets of contracts. A contract $c=(q, t, n)$ describes a decision $q \in \mathbb{R}_{+}$that is costly to the agent and a transfer $t \in \mathbb{R}$ that yields the agent some benefits upon acceptance. It includes the principal's identity $n \in \mathbb{N}$. Principals can determine transfers and decisions but their identity is fixed. Let $\mathcal{C}=\mathbb{R}_{+} \times \mathbb{R} \times \mathbb{N}$ denote the contract space. Let $C_{n}$ denote some set of contracts offered by principal $n$ and let $C=\bigcup_{n \in \mathbb{N}} C_{n}$ describe the total set of offered contracts.

Our paper extends the existing literature by assuming that an agent's utility upon accepting a contract can directly depend on the acceptance choices of the other agents. We assume that principals and agents do not have preferences over principals' identities as such. Identities are only required since agents need not be indifferent between two contracts specifying the same transfers and decisions as soon as principals attract different average types of agents.

Let the function $u: \Theta \times[\ell, h] \times \mathbb{R}_{+} \times \mathbb{R} \rightarrow \mathbb{R}$ describe agents' preferences where $u(\theta, y ; q, t)$ characterizes the utility of an agent of type $\theta$ who accepts contract $c=(q, t, n)$ if principal $n$ attracts agents of average type $y$. Function $u$ is at least twice partially differentiable in $y$, $q$, and $t$. We assume that $u_{q}<0, u_{t}>0, u_{q t}=0, u_{q q} \geq 0$, and $u_{t t} \leq 0$. Further, function $u(\theta, y ; q, \cdot)$ is unbounded above for all $(\theta, y, q)$ while $u(\theta, y ; \cdot, t)$ is unbounded below for all $(\theta, y, t)$. Externalities among agents are formalized as follows.

Definition 1 (Externality: Agents). We assume that $u_{y}>0$ for $y<\theta$ and $u_{y}=0$ for $y \geq \theta$. An agent's utility upon accepting a contract offered by some principal is increasing in the average type of agents attracted by the same principal, as long as this average type is lower 
than the agent's type. If an agent's type is weakly lower than the average type, he no longer experiences a negative externality. A low type's utility upon accepting a contract thus does not depend on the average type of agent attracted by the same principal.

To illustrate we repeatedly refer to our labor-market example from the introduction.

Example (cont'd): Consider the example discussed in the introduction. Firms offer contracts that specify a required education level $q$ and a base wage $t$. Part of the compensation package is also a team bonus $\gamma t$ with $\gamma>0$ which is exogenously fixed to the base salary. ${ }^{4}$ This team bonus is paid if the team is successful. By the O-ring production technology the team is successful if and only if both team members are highly productive. Let $b(y)$ be the probability of being matched with a high-productivity worker if $y$ is the average type of workers employed by firm $n$. We must have $b(\ell)=0$ and $b(h)=1$. In principle, the firm might use some internal mechanisms to affect worker matching, but our results hold as long as complete separation is impossible and $b$ is increasing in $y$. Then a worker's expected utility upon accepting contract $(q, t, n)$ is

$$
u(\theta, y ; q, t)= \begin{cases}t-q / \ell & \text { if } \theta=\ell \\ t-q / h+b(y) \gamma t & \text { if } \theta=h\end{cases}
$$

where $q / \theta$ are the usual type-specific costs of acquiring education.

We often refer to agents' preferences over decisions and transfers given that they are pooled with some fixed average type of agents. Define an agent's indifference curve $\bar{U}_{\theta}(y)$ in $(q, t)$ space as the set of all combinations of decisions and transfers which - if offered by a principal attracting agents of average type $y$ - yield an agent of type $\theta$ constant utility. Formally, this indifference curve is defined as

$$
\bar{U}_{\theta}(y)=\left\{(q, t) \in \mathbb{R}_{+} \times \mathbb{R}: u(\theta, y ; q, t) \equiv \bar{u}, \bar{u} \in \mathbb{R}\right\} .
$$

Indifference curves have slope $-u_{q}(\theta, y ; q, t) / u_{t}(\theta, y ; q, t)$, which does not depend on the average attracted type for low types, but might depend on the average type for high types. We assume that the slope of agents' indifference curves is decreasing in $\theta$ for all $y$. This is our version of the Spence-Mirrlees or single-crossing property. Requiring it to hold for all

\footnotetext{
${ }^{4}$ Thus, firms cannot use the team bonus as an additional instrument to screen workers. In Kosfeld and von Siemens (forthcoming), we analyze a particular application and show that this assumption can be relaxed without affecting our results concerning equilibrium existence.
} 
$y$ imposes a restriction: Changing the average attracted type must not affect the slope of the indifference curves of high types so as to upset the single-crossing property. Finally, we assume that the average attracted type $y$ has a monotone influence on the slope of high-type agents' indifference curves: $\frac{\partial}{\partial y}\left\{-u_{q}(h, y ; q, t) / u_{t}(h, y ; q, t)\right\}$ is either always weakly positive or always strictly negative. We normalize an agent's utility to zero in case he accepts no contract. We also assume that $u(\theta, \theta ; 0,0)=0$ for all $\theta$. If an agent is pooled with agents of his type and accepts a contract that specifies zero decision and zero transfer, he thus gets a utility equal to his outside option.

Our results are entirely driven by the externality among agents, so that principals' utility functions could be defined as usual. Yet, we show that including analogous externalities in the principals' utility function causes no problems. Let the function $v: \Theta \times[\ell, h] \times \mathbb{R}_{+} \times \mathbb{R} \rightarrow \mathbb{R}$ describe principals' preferences, where $v(\theta, y ; q, t)$ is principal $n$ 's utility per agent of type $\theta$ accepting contract $c=(q, t, n)$ if he attracts agents of average type $y$. Function $v$ is at least once partially differentiable in $q$ and $t$ with $v_{t}<0$ and $v_{q}=0$. We focus on adverse selection and the associated costs of separation and thus assume that the decision is non-productive. ${ }^{5}$ Function $v(\theta, y ; q, \cdot)$ is unbounded below for all $(\theta, y, t)$. Principals prefer high types so that $v(h, y ; q, t)>v(\ell, y ; q, t)$ for all $(y, q, t)$.

Definition 2 (Externality: Principals). We assume that $v_{y} \geq 0$ for $y<\theta$ and $v_{y}=0$ for $y \geq \theta$.

The presence of low types might reduce the principal's utility per attracted agent of high type, but as the definition shows this externality can also be zero without affecting our results. Let $\eta(\theta ; c, C)$ be the mass of agents of type $\theta$ accepting contract $c$ if set $C$ of contracts is offered. Principal $n$ who offers contracts $C_{n} \subseteq C$ then gets total utility

$$
\sum_{\theta \in \Theta} \sum_{c \in C_{n}} \eta(\theta ; c, C) v(\theta, y ; q, t)
$$

Principals get an outside option utility of zero if they do not attract any agents. We assume that $v(\theta, \ell ; 0,0)>0$ for all $\theta$ and $y$. A principal thus gets more than his outside option if he can attract agents with a contract that specifies a zero transfer. Together with the assumption on agents' outside option, this ensures that mutually beneficial contracting between

\footnotetext{
${ }^{5}$ In our labor market example the decision is education which - as in Spence (1973) - does not affect the worker's productivity. In case the decision is productive, first-best decisions might ensure separation so that there is no problem of adverse selection.
} 
principals and agents is possible.

Example (cont'd): The profit of a firm that employs a worker of type $\theta$ and promises base wage $t$ is $\theta-t$ if the workers team is not successful, and $(1+\gamma) \theta-(1+\gamma) t$ if the team is successful. We can vary the importance of our externality by varying $\gamma$, and we can do so without simultaneously affecting the problem of adverse selection in the labor market. If $\gamma$ is zero we get a simple screening version of Spence's job signalling model.

We focus on symmetric pure-strategy equilibria where all agents share the same type-dependent acceptance decisions and principals do not mix over their sets of offered contracts. Let $\mathcal{P}(\mathcal{C})$ be the power set of contract space $\mathcal{C}$. Since we only consider symmetric equilibria we suppress indexation for an agent's identity in the following. An agent's completely specified strategy is an acceptance rule $a: \Theta \times \mathcal{C} \times \mathcal{P}(\mathcal{C}) \rightarrow[0,1]$ where $a(\theta ; c, C) \in[0,1]$ is the probability with which he accepts contract $c \in C$ if he is of type $\theta$. An acceptance rule can only assign a positive acceptance probability to contracts that are offered. For all $C \in \mathcal{C}$ and $\theta \in \Theta, a(\theta ; c, C)=0$ whenever $c \notin C$.

Agents' acceptance rules define the mass $\eta(\theta ; c, C)$ of agents of type $\theta$ accepting $c \in C$. Let

$$
A(n ; C)=\sum_{\theta \in \Theta} \sum_{c \in C_{n}} \eta(\theta ; c, C)
$$

be the total mass of agents attracted by principal $n$ if he offers contracts $C_{n} \subseteq C$. Unless $A(n ; C)$ equals zero the average type $y(n ; C)$ of agents attracted by principal $n$ is directly determined as

$$
y(n ; C)=\frac{1}{A(n ; C)} \sum_{\theta \in \Theta} \sum_{c \in C_{n}} \eta(\theta ; c, C) \theta .
$$

If $A(n ; C)$ equals zero, $y(n ; C)$ is not pinned down by the agents' acceptance decisions. In our setting this does not cause any problems: If an agent accepts a contract offered by some principal who attracts no other agents, his type determines the average type of agents attracted by the principal. ${ }^{6}$ As a single agent has mass zero he cannot influence the average agent attracted by a principal as soon as this principal draws a positive mass of agents.

In the presences of externalities an agent's utility might depend on the acceptance decisions of the other agents. We account for this as follows in our definition of equilibrium. Extending

\footnotetext{
${ }^{6}$ In contrast to a Walrasian approach (cf. introduction), we thus have no problems concerning the specification of out-of-equilibrium beliefs.
} 
the notion of a competitive equilibrium from Rothschild and Stiglitz (1976) we define an equilibrium as an equilibrium set of offered contracts plus agents' equilibrium acceptance rules. An equilibrium set $C^{*}$ of offered contracts must satisfy two conditions. First, each principal gets at least zero utility in equilibrium. ${ }^{7}$ Second, no principal $\tilde{n}$ can enter the market with a menu of contracts $C_{\tilde{n}}$ that can attract a strictly positive mass of agents while yielding positive overall utility. Perfect competition is thus formalized via a no-market-entry condition. Concerning the agents we require their equilibrium acceptance decisions $a^{*}$ to form a Bayesian equilibrium for all sets $C \subseteq \mathcal{C}$ of offered contracts: Acceptance decisions maximize the agent's utility given the distribution $\eta^{*}$ of agents across principals, and this distribution is consistent with the distribution of agents' types and their corresponding acceptance decisions.

\section{$2.2 \quad$ Further Definitions and a Refinement}

One of our main results is that there must be separation in any competitive equilibrium. Since principals' identities as such are irrelevant, the model is silent on which principal offers which contract in equilibrium. However, agents' and principals' utility functions uniquely pin down the decisions and transfers of contracts that are accepted by high and low types. These contracts include transfers $\left(t_{\ell}, t_{h}\right)$ and decision $q_{h}$ that are implicitly defined by

$$
\begin{aligned}
v\left(\ell, y ; 0, t_{\ell}\right) & =0 \\
v\left(h, h ; q_{h}, t_{h}\right) & =0 \\
u\left(\ell, y ; q_{h}, t_{h}\right) & =u\left(\ell, y ; 0, t_{\ell}\right) .
\end{aligned}
$$

As a low type's utility does not depend on the average attracted type, the choice of $y$ in (5) and (7) is irrelevant. We show in the Appendix that our assumptions on $v$ and $u$ guarantee the existence of a unique, finite, and strictly positive solution to (5) to (7) with $t_{h}>t_{\ell}$. We can define the sets of contracts

$$
\begin{aligned}
& C_{\ell}=\left\{(q, t, n) \in \mathcal{C}: q=0 \text { and } t=t_{\ell}\right\} \\
& C_{h}=\left\{(q, t, n) \in \mathcal{C}: q=q_{h} \text { and } t=t_{h}\right\}
\end{aligned}
$$

with generic elements $c_{\ell} \in C_{\ell}$ and $c_{h} \in C_{h}$.

Definition 3 (Best Separating Equilibrium). In a best separating equilibrium principals offer contracts from both sets $C_{\ell}$ and $C_{h}$. Moreover, the following properties hold:

${ }^{7}$ We thus allow for cross-subsidization since principals can offer several contracts. However, the possibility for cross-subsidization does not drive any of our results. 
1. All agents accept some contracts. Agents of type $\ell$ only accept contracts from $C_{\ell}$ while agents of type $h$ only accept contracts from $C_{h}$.

2. Agents separate across principals according to their type. There are no contracts $\{c, \tilde{c}\} \subseteq$ $C_{n}^{*}$ offered by some principal $n$ with $a^{*}\left(h ; c, C^{*}\right)>0$ and $a^{*}\left(\ell ; \tilde{c}, C^{*}\right)>0$.

Due to externalities there can be multiple equilibria at the acceptance stage. The following refinement rules out competitive equilibria that exist only because agents re-coordinate on acceptance decisions once otherwise irrelevant new contracts are offered.

Refinement (No Switch). Consider an equilibrium set $C^{*}$ of offered contracts. Suppose a new principal $\tilde{n}$ enters the market with a set of contracts $C_{\tilde{n}}$ that cannot attract any agents so that $a^{*}\left(\theta ; c, C^{*} \cup C_{\tilde{n}}\right)=0$ for all $\theta \in \Theta, c \in C_{\tilde{n}}$. Then agents must keep their old acceptance decisions, $a^{*}\left(\theta ; c, C^{*} \cup C_{\tilde{n}}\right)=a^{*}\left(\theta ; c, C^{*}\right)$ for all $c \in C^{*}$.

\section{Results}

\subsection{Existence of Best Separating Equilibria (Large Externality)}

Before we come to our main result (Proposition 1), we first derive conditions for the existence of a best separating equilibrium if principals do not offer contracts that are not accepted in equilibrium. In this case, the following lemma shows that the utility loss of high types from being pooled with only low types must exceed the minimum screening costs high types have to incur in order to ensure separation in equilibrium. The lemma prepares the ground for our main result on arbitrarily small externalities. All proofs can be found in the appendix.

Lemma 1 (Best Separating Equilibrium - Large Externality). There exists a best separating equilibrium for all $\mu_{0} \in(0,1)$ in which principals offer only contracts in $C_{\ell} \cup C_{h}$ if and only if $u\left(h, h ; q_{h}, t_{h}\right) \geq u\left(h, \ell ; 0, t_{h}\right)$.

Contrary to the situation without externalities, market entry with a pooling contract need not be profitable even if the fraction of agents of the low type is arbitrarily small. Indeed, in one equilibrium at the acceptance stage all agents optimally accept the new pooling contract. But in another equilibrium the high types do not to accept the new contract because the new principal only attracts low types. Since the new principal would then make losses, he does not enter the market. Note that this result is not driven by some arbitrary specification of out-of-equilibrium beliefs. If a new principal does enter the market, he does attract all low types so that these agents' acceptance decisions pin down the average attracted type. 
Instead, it is the multiplicity of Bayesian equilibria at the acceptance stage which solves the equilibrium existence problem.

Example (cont'd): In our example the best separating contracts specify $t_{\ell}=\ell$ for lowproductivity workers, and $t_{h}=h$ and $q_{h}=\ell(h-\ell)$ for high-productivity workers. These contracts can form a competitive equilibrium with Pareto-dominant separation for all $\mu_{0}$ if and only if

$$
\gamma h \geq \frac{\ell(h-\ell)}{h} .
$$

Choosing their separating contract high-productivity workers can secure themselves the bonus $\gamma t$, but they incur screening costs $q_{h} / h=\ell(h-\ell) / h$. By accepting the pooling contract they save the screening costs, but as they are matched with low-productivity workers they loose the bonus. If the relation $\gamma$ of bonus to base salary - which in our example corresponds to a measure of the externality among agents - is sufficiently large, there always exists a best separating equilibrium. As shown by Rothschild and Stiglitz (1976) this condition cannot be fulfilled if the externality $\gamma$ disappears.

\subsection{Existence of Best Separating Equilibria (Arbitrarily Small Externality)}

Lemma 1 describes conditions for the existence of a best separating equilibrium in which principals do not offer any contracts that are not in $C_{\ell}$ or $C_{h}$. We now demonstrate that an arbitrarily small externality is sufficient to guarantee equilibrium existence if principals can offer contracts that are never accepted in equilibrium. The sole purpose of these preemptive contracts is to prevent market entry.

The argument runs as follows. Consider a principal who attracts only low types in equilibrium. Suppose this principal also offers some contract that would be very attractive for high types if he attracted only high types - but in equilibrium this contract is not accepted as the principal only attracts low types. Now if a principal enters the market and draws all low types, the old principal offering the preemptive contract attracts no agents any more. He thus suddenly becomes very attractive for high types. However, if the new principal cannot draw any high types, he gets negative utility and there is no market entry. ${ }^{8}$

\footnotetext{
${ }^{8}$ The argument resembles the line of reasoning in Riley (1979) who allows firms to offer new contracts and thereby to skim off the good types after market entry. In our model such contracts are already offered in equilibrium.
} 
To analyze preemptive contracts, let $\Gamma$ be the set of all combinations of transfers and decisions that do not attract any high types if offered by a principal who currently attracts only low types. $\Gamma$ contains all $(q, t)$ that satisfy

$$
\begin{aligned}
u\left(\ell, y ; q_{\ell}, t_{\ell}\right) & \geq u(\ell, y ; q, t) \\
u\left(h, h ; q_{h}, t_{h}\right) & \geq u(h, \ell ; q, t) .
\end{aligned}
$$

As a low type's utility does not depend on the average attracted type, the choice of $y$ in (11) is irrelevant. Because preemptive contracts do not attract any agents in equilibrium, they cannot cause any losses. No constraint concerning the utility of the offering principal is needed. Define

$$
U_{p}=\sup _{(q, t) \in \Gamma}\{u(h, h ; q, t)\}
$$

as the supremum of the utility which high types can get if they accept a preemptive contract which is offered by a principal who - after market entry - attracts no agents. There is the following result.

Lemma 2 (Supremum Utility Preemptive Contracts). Consider the supremum utility $U_{p}$ that high-type agents can get by accepting a preemptive contract after market entry.

1. If $\frac{\partial}{\partial y}\left\{-u_{q}(h, y ; q, t) / u_{t}(h, y ; q, t)\right\} \geq 0$ then $U_{p}=u\left(h, h ; q_{p}, t_{p}\right)$, where $\left(q_{p}, t_{p}\right)$ is implicitly defined by $u\left(\ell, y ; 0, t_{\ell}\right)=u\left(\ell, y ; q_{p}, t_{p}\right)$ and $u\left(h, h ; q_{h}, t_{h}\right)=u\left(h, \ell ; q_{p}, t_{p}\right)$.

2. If $\frac{\partial}{\partial y}\left\{-u_{q}(h, y ; q, t) / u_{t}(h, y ; q, t)\right\}<0$ then $U_{p}=+\infty$.

Figure 1 illustrates Lemma 2. Set $\Gamma$ is the area under the two indifference curves $\bar{U}_{\ell}(y)$ and $\bar{U}_{h}(\ell)$ for some $y$ (since the average attracted type is irrelevant for low types). If increasing the average attracted type does not flatten the indifference curves of high types, the supremum utility $U_{p}$ is finite and is attainable by accepting a preemptive contract with decision and transfer $\left(q_{p}, t_{p}\right)$. But if increasing $y$ flattens the indifference curves of high types, moving up along $\bar{U}_{h}(\ell)$ increases the high types' utility without violating any constraint. Figure 1 shows that in this case $U_{p}$ equals plus infinity. It is now possible to characterize sufficient and necessary conditions for the existence of a best separating equilibrium.

Proposition 1 (Best Separating Equilibrium - Arbitrarily Small Externality).

1. There exists a best separating equilibrium for all $\mu_{0} \in(0,1)$ if and only if $U_{p} \geq u\left(h, \ell ; 0, t_{h}\right)$.

2. If $\frac{\partial}{\partial y}\left\{-u_{q}(h, y ; q, t) / u_{t}(h, y ; q, t)\right\}<0$ then $U_{p}=+\infty$ even if externalities are arbitrarily small. In this case equilibrium existence is guaranteed. 


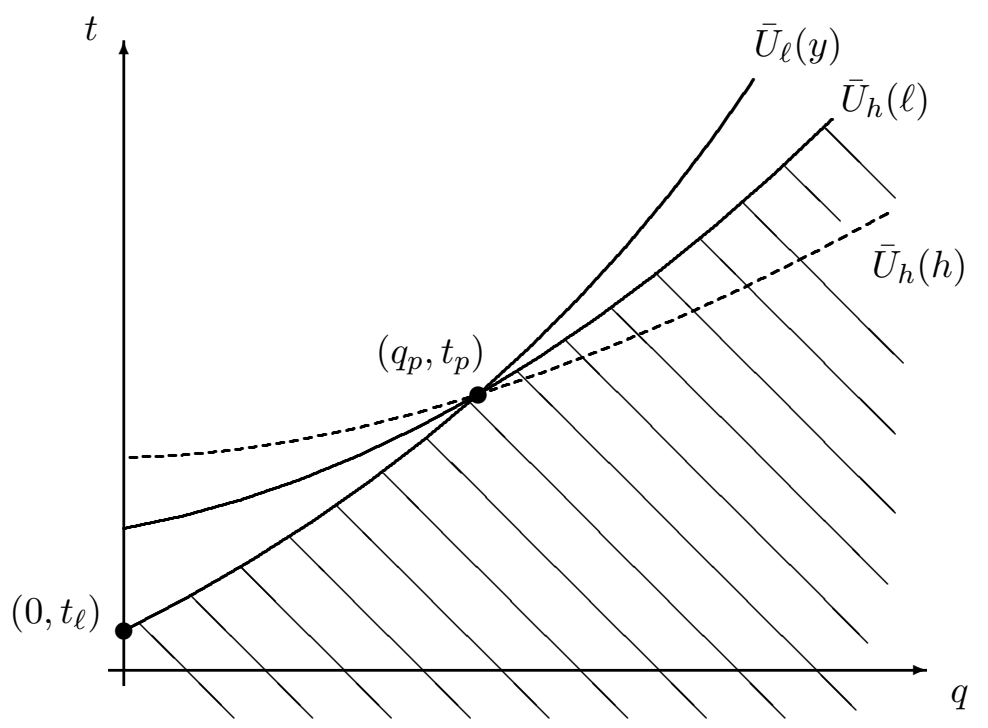

Figure 1: Set $\Gamma$ is the shaded area.

Lemma 2 and Proposition 1 imply that arbitrarily small externalities can ensure equilibrium existence. In the following we show with the help of our example that the required flattening of the high types' indifference curves is well plausible.

Example (cont'd): In our example a high-productivity worker is indifferent to - and thus optimally rejects - any preemptive contract that specifies for education level $\tilde{q}$ a base salary $\tilde{t}=t+\tilde{q} / h$ where

$$
t=h(1+\gamma)-\frac{1}{\theta} \ell(h-\ell)
$$

Among these contracts we look for a preemptive contract that satisfies two conditions. First, the preemptive contract is not accepted by low-productivity workers. Using these workers' equilibrium utility yields the following condition

$$
\tilde{q} \geq \frac{\ell h}{h-\ell}\left(h(1+\gamma)-\ell-\frac{1}{h}(h-\ell)\right) .
$$

Second, the preemptive contract must attract all high-productivity workers in case there is market entry. If the pooling contract only attracts low-productivity workers, a high-productivity worker who joins the new firm never gets the bonus and thus does not earn more than utility h. He prefers the preemptive contract in case

$$
\tilde{q} \geq \frac{h}{\gamma}\left(h(1+\gamma)-\frac{1}{h} \ell(h-\ell)\right) .
$$

Market entry can thus be prevented by a preemptive contract that specifies an educational level sufficiently large so as to satisfy both (15) and (16). Such a contract can be easily found 
for any level of externality $\gamma$, thus an arbitrarily small externality is sufficient to guarantee equilibrium existence.

\subsection{Description of Equilibrium Outcome}

So far we have focused on the sufficient and necessary conditions under which externalities ensure the existence of a best separating equilibrium. We now proceed to show that whenever there exists a competitive equilibrium in pure strategies, it must be a best separating equilibrium. The main step is to show that there cannot be pooling in equilibrium.

Lemma 3 (No Pooling). In any competitive equilibrium there are no contracts $\{c, \hat{c}\} \subseteq C_{n}^{*}$ offered by some principal $n$ with $a^{*}\left(\ell ; c, C^{*}\right)>0$ and $a^{*}\left(h ; \hat{c}, C^{*}\right)>0$.

Adapting the arguments by Rothschild and Stiglitz (1976) it is now easy to show that any competitive equilibrium must be a best separating equilibrium. Since only low types exert an externality on high types, low types behave as if there were no externalities at all. In any competitive equilibrium they must thus get their contracts from set $C_{\ell}$. If high types do not get their contracts from $C_{h}$, then a new principal can enter the market and offer a contract that brings these agents closer to the best separating equilibrium. This contract is designed so as to never attract low types. High types can then be certain that they will be either alone or among themselves whenever they accept the new contract. A strictly positive mass of high types is thus attracted while low types stick to their old contract choices. The new contract then yields the principal strictly positive utility, and there is market entry.

Proposition 2 (Description and Existence of Competitive Equilibrium).

1. Any competitive equilibrium is a best separating equilibrium.

2. A competitive equilibrium exists if and only if $U_{p} \geq u\left(h, \ell ; 0, t_{h}\right)$.

As in Rothschild and Stiglitz (1976) any competitive equilibrium is characterized by Paretodominant separation. Although externalities facilitate equilibrium existence, they do not affect equilibrium predictions. Moreover, sufficient and necessary conditions for the existence of a best separating equilibrium are sufficient and necessary conditions for the existence of any competitive equilibrium.

\section{Discussion}

In this paper we show that particular negative externalities among agents can mitigate the equilibrium existence problem in competitive markets with adverse selection. Already in a 
standard adverse selection setting there are externalities among agents: In the labor market example the presence of workers with low-productivity prevents firms from offering high wages to workers of high-productivity without requiring them to acquire some minimum education that ensures separation. The externalities we have in mind are more direct as they arise once agents contract with the same principal. It is crucial that these externalities lie beyond the control of principals: Suppose a principal can credibly guarantee to protect high types from low types - for example by structural means that separate types within the organization, or by committing to precisely specified contractual terms. Since this makes pooling contracts attractive again, there is market entry and the equilibrium existence problem continues to exist. However, it is not obvious whether principals can in fact credibly commit to eliminate externalities among their agents. For example, having separate plants for workers of different productivity might be prohibitively expensive or impossible given the production technology.

Our model does not consider positive externalities, where low types want to be pooled with hight types but high types do not care about the presence of low types. This could have the following consequences. Since positive externalities do not affect the high types' acceptance decisions, they do not influence the attractiveness of a pooling contract for high types. The latter determines the stability of a best separating equilibrium, thus weak externalities have no effect on the conditions for equilibrium existence. However, low types now want to be pooled with high types. If externalities are strong, it might thus no longer be possible to skim off high types from a pooling contract since low types always follow. Strong positive externalities might thus render the existence of pooling equilibria possible. Beyond the scope of the present paper, a complete characterization of the consequences of positive and negative externalities in competitive markets with adverse selection thus remains a fruitful topic for further research.

\section{Appendix (Proofs)}

\section{Definition of a Best Separating Equilibrium}

$v(\ell, y ; 0,0)>0$ and $v(\ell, y ; 0, \cdot)$ is unbounded below. Then $v_{t}<0$, continuity of $v$, and the intermediate value theorem imply that there exists a unique, finite, and strictly positive $t_{\ell}$ satisfying (5). By the same argument there exists a unique, finite, and strictly positive $t_{h}$ satisfying (6) where $v_{q}=0$ implies that $t_{h}$ does not depend on $q_{h}$. 
$v(h, y ; q, t)>v(\ell, y ; q, t)$ for all $(y, q, t)$. Then $v\left(h, h ; q_{h}, t_{\ell}\right)>v\left(\ell, h ; q_{h}, t_{\ell}\right)=0$ where the last equality follows from $v_{q}=0$ and $v_{y}(\ell, y ; q, t)=0$ for all $(y, q, t)$. This yields $t_{h}>t_{\ell}$ so that $u\left(\ell, y ; 0, t_{h}\right)>u\left(\ell, y ; 0, t_{\ell}\right)$ since $u_{t}>0$. Function $u\left(\ell, y ; \cdot, t_{h}\right)$ is unbounded below. Then $u_{q}<0$, continuity of $u$, and the intermediate value theorem imply the existence of a unique, finite, and strictly positive $q_{h}$ satisfying (7).

\section{Proof of Lemma 1}

We first show that agents get more than their outside option in a best separating equilibrium. This holds directly for low types since $t_{\ell}>0, u_{t}>0$, and $u(\ell, y ; 0,0)=0$ for all $y$. By definition $u\left(\ell, h ; q_{h}, t_{h}\right)=u\left(\ell, h ; 0, t_{\ell}\right)$. This yields $u\left(h, h ; q_{h}, t_{h}\right)>u\left(h, h ; 0, t_{\ell}\right)$ because $q_{h}>0$ and the single crossing property holds for all $y$. However, $u\left(h, h ; 0, t_{\ell}\right)>0$ follows from $t_{\ell}>0$, $u_{t}>0$, and $u(h, h ; 0,0)=0$. In equilibrium high types thus get more than their outside option. We next show our main result.

\section{Part 1: Sufficiency}

Consider a best separating equilibrium in which principals only offer contracts in $C_{\ell} \cup C_{h}$. By definition this equilibrium cannot be upset by a principal who enters the market and then attracts only one type of agents.

Suppose principal $\tilde{n}$ enters the market with contracts $\tilde{c}_{\ell}=\left(\tilde{q}_{\ell}, \tilde{t}_{\ell}, \tilde{n}\right)$ and $\tilde{c}_{h}=\left(\tilde{q}_{h}, \tilde{t}_{h}, \tilde{n}\right)$ for type $\ell$ and $h$. As otherwise type $\ell$ optimally rejects, $u_{y}(\ell, y ; q, t)=0$ and $u_{q}<0$ imply $\tilde{t}_{\ell}>t_{\ell}$. Because they do not care for the average attracted type, type $\ell$ 's acceptance decisions do not depend on the behavior of type $h$. If $a^{*}\left(\ell ; \tilde{c}_{\ell}, C^{*} \cup C_{\tilde{n}}\right)$ must be strictly positive in one, then $\tilde{n}$ attracts all type $\ell$ in any equilibrium at the acceptance stage.

$v_{y}(\ell, y ; q, t)=0, v_{q}=0$, and $\tilde{t}_{\ell}>t_{\ell}$ imply $v\left(\ell, y ; \tilde{q}_{\ell}, \tilde{t}_{\ell}\right)<0$. Principal $\tilde{n}$ makes losses unless he can attract $h$. The proof proceeds to show that for every equilibrium at the acceptance stage with $a^{*}\left(h ; \tilde{c}_{h}, C^{*} \cup C_{\tilde{n}}\right)>0$ in which the principal breaks even, there exists another equilibrium with $a^{*}\left(h ; \tilde{c}_{h}, C^{*} \cup C_{\tilde{n}}\right)=0$. Given these acceptance decisions principal $\tilde{n}$ makes losses and thus does not enter the market.

Suppose $a^{*}\left(h ; \tilde{c}_{h}, C^{*} \cup C_{\tilde{n}}\right)>0$ and principal $\tilde{n}$ makes no losses: if $y_{\tilde{n}}$ is his average attracted type, $v\left(h, y_{\tilde{n}} ; \tilde{q}_{h}, \tilde{t}_{h}\right)>0$ holds. With $v_{t}<0, u_{t}>0, u_{q}<0$, and $v_{q}=0$, this implies that type $h$ who accept $\tilde{c}_{h}$ cannot get more than $u\left(h, y_{\tilde{n}} ; 0, \tilde{t}_{h}\right)$ where $\tilde{t}_{h}$ solves $v\left(h, y_{\tilde{n}} ; 0, \tilde{t}_{h}\right)=0$. 
Take $t_{h}$ from Definition 3. Decisions are not productive so that $v_{y}(h, y ; q, t) \leq 0$ and $y_{\tilde{n}} \leq h$ imply $t_{h} \geq \tilde{t}_{h}$. Then $a^{*}\left(\ell ; \tilde{c}_{\ell}, C^{*} \cup C_{\tilde{n}}\right)=1$ and $a^{*}\left(h ; \tilde{c}_{h}, C^{*} \cup C_{\tilde{n}}\right)=0$ with $y_{\tilde{n}}=\ell$ form an equilibrium at the acceptance stage. Type $\ell$ act optimally by the above arguments. Further, type $h$ who accept $\tilde{c}_{h}$ get less than $u\left(h, \ell ; 0, \tilde{t}_{h}\right)$ and thus less than $u\left(h, \ell ; 0, t_{h}\right)$. They get $u\left(h, h ; q_{h}, t_{h}\right)$ by accepting $c \in C_{h}$. As $u\left(h, h ; q_{h}, t_{h}\right) \geq u\left(h, \ell ; 0, t_{h}\right)$ they choose $c_{h}$.

\section{Part 2: Necessity}

Take a best separating equilibrium and consider a sequence $\left\{\mu_{k}\right\}_{k \in \mathbb{N}}$ of prior probabilities with $\mu_{k}<1$ for all $k \in \mathbb{N}$ but $\lim _{k \rightarrow \infty} \mu_{k}=1$. Define $y_{k}=\ell+\mu_{k}(h-\ell)$ and $\epsilon_{k}=1 / k$. Given $\mu_{k}$ suppose a principal $n$ enters the market with a pooling contract $c_{k}=\left(0, t_{k}-\epsilon_{k}, n\right)$ where $t_{k}$ is implicitly defined by $\mu_{k} v\left(h, y_{k} ; 0, t_{k}\right)+\left(1-\mu_{k}\right) v\left(\ell, y_{k} ; 0, t_{k}\right)=0$. He thus gets strictly positive utility if he can attract all agents. Then $v_{q}=0$ and continuity of $v$ imply $\lim _{k \rightarrow \infty} t_{k}=t_{h}$. If $u\left(h, h ; q_{h}, t_{h}\right)<u\left(h, \ell ; 0, t_{h}\right)$ then continuity of $u$ implies that there exists a $K \in \mathbb{N}$ so that for all $k \geq K$ we have $u\left(h, \ell ; 0, t_{k}\right)>u\left(h, h ; q_{h}, t_{h}\right)$. In the following take some $\mu_{k}$ with $k \geq K$.

Given prior probability $\mu_{k}$ and market entry by principal $n$, type $\ell$ accept $c_{k}$ since $t_{k}>t_{\ell}$. As $u\left(h, \ell ; 0, t_{k}\right)>u\left(h, h ; q_{h}, t_{h}\right)$ type $h$ prefer $c_{k}$ to any $c_{h} \in C_{h}$ even if $y\left(n ; C^{*} \cup c_{k}\right)=\ell$. The single-crossing property and $q_{h}>0$ imply that high types prefer contract $c_{h} \in C_{h}$ to any contract $c_{\ell} \in C_{\ell}$ even if the principal who offers $c_{\ell}$ does not attracting any low types. Only contracts in $C_{\ell} \cup C_{h}$ are offered. Transitivity implies $a^{*}\left(h ; c_{k}, C^{*} \cup c_{k}\right)=1$ and $a^{*}\left(\ell ; c_{k}, C^{*} \cup c_{k}\right)=1$ in any equilibrium at the acceptance stage. As he thereby gets strictly positive utility, principal $n$ enters the market.

Q.E.D.

\section{Proof of Lemma 2}

$\Gamma$ is the area under indifference curves $\bar{U}_{\ell}(y)$ and $\bar{U}_{h}(\ell)$ which correspond to utility levels $u\left(\ell, y ; q_{\ell}, t_{\ell}\right)$ and $u\left(h, h ; q_{h}, t_{h}\right)$. By definition $\bar{U}_{\ell}(y)$ contains $\left(0, t_{\ell}\right)$ and $\left(q_{h}, t_{h}\right)$ since $(7)$ does not depend on the particular $y$. Take an indifference curve $\bar{U}_{h}^{\prime}(\ell)$ which corresponds to utility level $u\left(h, \ell ; q_{h}, t_{h}\right)$ and thus contains $\left(q_{h}, t_{h}\right)$. By the single-crossing property $\bar{U}_{h}^{\prime}(\ell)$ is flatter than $\bar{U}_{\ell}(y)$ and thus runs above $\bar{U}_{\ell}(y)$ to the left of $\left(q_{h}, t_{h}\right)$. It intersects the $t$-axis at $\tilde{t}>t_{\ell}$ where $\tilde{t}$ solves $u(h, \ell ; 0, \tilde{t})=u\left(h, \ell ; q_{h}, t_{h}\right) . \bar{U}_{h}(\ell)$ which corresponds to utility level $u\left(h, h ; q_{h}, t_{h}\right)>u\left(h, \ell ; q_{h}, t_{h}\right)$ runs above $\bar{U}_{h}^{\prime}(\ell)$. Set $\Gamma$ is thus as illustrated in Figure 1.

We look for the supremum $U_{p}$ as defined in (13). First, take any $(q, t)$ so that both (11) and (12) are slack. Then one can increase the transfer $t$ without violating any constraint so 
that $U_{p}>u(h, h ; q, t)$ for such $(q, t)$. Second, take any $(q, t)$ so that (11) is binding while (12) is slack. Consider marginal changes $d t, d q>0$ with $d t / d q=-u_{q}(\ell, y ; q, t) / u_{t}(\ell, y ; q, t)$. This keeps (11) satisfied while the single-crossing property implies that (12) remains satisfied and $u(h, h ; q+d q, t+d t)>u(h, h ; q, t)$. Thus, $U_{p}>u(h, h ; q, t)$ for such $(q, t)$. Third, take the $\left(q_{p}, t_{p}\right)$ so that by definition both (11) and (12) are binding. Consider marginal changes $d t, d q>0$ with $d t / d q=-u_{q}\left(h, \ell ; q_{p}, t_{p}\right) /\left(u_{t}\left(h, \ell ; q_{p}, t_{p}\right)\right.$. This keeps (12) satisfied while slackening (11) by the single-crossing property. There are then two cases.

First, suppose $\frac{\partial}{\partial y}\left\{-u_{q} / u_{t}\right\} \geq 0$. Then $u\left(h, h ; q_{p}, t_{p}\right) \geq u\left(h, h ; q_{p}+d q, t_{p}+d t\right)$ so that contracts with $\left(q_{p}, t_{p}\right)$ provide type $h$ with the maximum utility $U_{p}=u\left(h, h ; q_{p}, t_{p}\right)$. Second, suppose $\frac{\partial}{\partial y}\left\{-u_{q} / u_{t}\right\}<0$. Then $u\left(h, h ; q_{p}, t_{p}\right)<u\left(h, h ; q_{p}+d q, t_{p}+d t\right)$. Because one can find similar contract adjustments for all $(q, t)$ such that (11) is slack while (12) is binding, there exists an infinite sequence $\left\{\left(q_{k}, t_{k}\right)\right\}_{k \in \mathbb{N}}$ where $\lim _{k \rightarrow \infty} u\left(h, h ; q_{k}, t_{k}\right)=+\infty$ and $\left(q_{k}, t_{k}\right) \in \Gamma$ for all $k \in \mathbb{N}$. In this case we have $U_{p}=+\infty$.

Q.E.D.

\section{Proof of Proposition 1}

Consider a best separating equilibrium in which principal $n$ offers a contract $c_{\ell} \in C_{\ell}$ that attracts type $\ell$. Suppose $n$ also offers a contract $c_{n} \notin C_{\ell} \cup C_{h}$ while $c_{n} \in \Gamma$. This contract $c_{n}=\left(q_{n}, t_{n}, n\right)$ is not accepted in equilibrium, but it changes the conditions under which a newly offered contract $\tilde{c}_{h}$ can attract type $h$. Contract $\tilde{c}_{h}$ need not draw type $h$ if and only if they have a better option in $C^{*}$. Type $h$ who accept $c_{n}$ can now get $u\left(h, h ; q_{n}, t_{n}\right)$ once $n$ no longer attracts any type $\ell$. There exists $c_{n} \in \Gamma$ so that they reject $\tilde{c}_{h}$ for all $\mu_{0} \in(0,1)$ if and only if $U_{p} \geq u\left(h, \ell ; t_{h}, 0, \tilde{n}\right)$.

Q.E.D.

\section{Proof of Lemma 3}

Consider a competitive equilibrium in which principal $n$ offers one or several contracts and attracts all types of agents. Let $y^{*}\left(n ; C^{*}\right) \in(\ell, h)$ be the average type of agent he attracts in equilibrium. The proof proceeds in two steps.

\section{Part 1: Strictly Positive Utility with Low Types}

Let $c=(q, t, n)$ be a contract offered by $n$ that attracts $\ell$ while $v(\ell, y ; q, t)>0$. Suppose a new principal $\tilde{n}$ enters the market with contract $\tilde{c}=(q, t+\epsilon, \tilde{n})$ with $\epsilon>0$. Then $u_{t}>0$ and $u_{y}=0$ for type $\ell$ imply $u(\ell, \tilde{y} ; q, t+\epsilon)>u(\ell, y ; q, t)$ for any $\tilde{y}$ and $y$. Consequently, $a^{*}\left(\ell ; \tilde{c}, C^{*} \cup \tilde{c}\right)=1$ and contract $\tilde{c}$ can attract at least all type $\ell$ in any equilibrium at the 
acceptance stage. Furthermore, $v_{y}(\ell, y ; q, t)=0$ and $v(\ell, y ; q, t)>0$ imply $v(\ell, \ell ; q, t+\epsilon)>0$ for small $\epsilon$. Finally, $v(h, y ; q, t+\epsilon)>v(\ell, y ; q, t+\epsilon)$ for all $y$ so that $\tilde{c}$ always yields principal $\tilde{n}$ strictly positive utility no matter what agents are attracted. As $\tilde{c}$ attracts at least agents of type $\ell$ the original situation cannot form an equilibrium.

\section{Part 2: Weakly Negative Utility with Low Types}

Now suppose there is no $c \in C_{n}^{*}$ that attracts $\ell$ and $v\left(\ell, y^{*}\left(n ; C^{*}\right) ; q, t\right)>0$. As principal $n$ otherwise gets strictly negative utility in equilibrium, there must exist $\hat{c}=(\hat{q}, \hat{t}, n) \in C_{n}^{*}$ that attracts $h$ and $v\left(h, y^{*}\left(n ; C^{*}\right) ; \hat{q}, \hat{t}\right) \geq 0$. Suppose a new principal $\tilde{n}$ enters the market and offers contract $\tilde{c}=(\hat{q}, \hat{t}-\epsilon, \tilde{n})$ with $\epsilon>0$.

Together with the optimality of the original contract choice, $u_{t}>0$ and $u_{y}(\ell, y ; q, t)=0$ imply $u(\ell, y ; q, t) \geq u(\ell, y ; \hat{q}, \hat{t})>u(\ell, \tilde{y} ; \hat{q}, \hat{t}-\epsilon)$ for any $y$ and $\tilde{y}$. Then $a^{*}\left(\ell ; \tilde{c}, C^{*} \cup \tilde{c}\right)=0$ so that $\tilde{c}$ never attracts type $\ell$ in any equilibrium at the acceptance stage. Because we restrict attention to symmetric acceptance decisions, there are two cases.

First, suppose $\tilde{c}$ attracts nobody. Then Refinement (No Switch) requires $a^{*}\left(\theta ; c, C^{*} \cup \tilde{c}\right)=$ $a^{*}\left(\theta ; c, C^{*}\right)$ for all $\theta \in \Theta, c \in C^{*}$. Type $h$ get utility $u\left(h, y^{*}\left(n ; C^{*}\right) ; \hat{q}, \hat{t}\right)$ in equilibrium. Then $y^{*}\left(n ; C^{*}\right)<h$ and $u_{y}(h, y ; q, t)<0$ imply $u(h, h ; \hat{q}, \hat{t}-\epsilon)>u\left(h, y^{*}\left(n ; C^{*}\right) ; \hat{q}, \hat{t}\right)$ for small $\epsilon$. An agent who alone accepts $\tilde{c}$ determines the average type attracted by $\tilde{n}$. Rejecting $\tilde{n}$ thus cannot be optimal for type $h$. Second, suppose $\tilde{c}$ attracts type $h$. In both cases principal $\tilde{n}$ then gets strictly positive utility from entering the market because $v_{t}<0, v_{y}(h, y ; \hat{q}, \hat{t}) \geq 0$, and $y^{*}\left(n, C^{*}\right)<h$ imply $v(h, h ; \hat{q}, \hat{t}-\epsilon)>v\left(h, y^{*}\left(n, C^{*}\right) ; \hat{q}, \hat{t}\right) \geq 0$.

Q.E.D.

\section{References}

Bester, H. (1985): "Screening vs. Rationing in Credit Markets with Imperfect Information," American Economic Review, 75(4), 850-855.

Cho, I.-K., And D. M. Kreps (1987): "Signalling Games and Stable Equilibria," Quarterly Journal of Economics, 102, 179-221.

Dalmazzo, A. (2002): "Technological Complexity, Wage Differentials and Unemployment," Scandinavian Journal of Economics, 104(4), 515-530. 
Dubey, P., and J. Geanakoplos (2002): "Competitive Pooling: Rothschild-Stiglitz Reconsidered," Quarterly Journal of Economics, 117(4), 1529-1570.

FABEL, O. (2004): "Spinoffs of Entrepreneurial Firms: An O-Ring Approach," Journal of Institutional and Theoretical Economics, 160, 416-438.

Gale, D. (1992): "A Walrasian Theory of Markets with Adverse Selection," Review of Economic Studies, 59(2), 229-255.

Hellwig, M. (1987): "Some Recent Developments in the Theory of Competition in Markets with Adverse Selection," European Economic Review, 31, 319-325.

Jones, C. I. (2008): "Intermediate Goods and Weak Links: A Theory of Economic Development," mimeo U.C. Berkeley.

Kosfeld, M., and F. von Siemens (forthcoming): "Worker Self-Selection and the Profits from Cooperation," Journal of the European Economic Association (Papers and Proceedings).

Kremer, M. (1993): "The O-Ring Theory of Economic Development," Quarterly Journal of Economics, 108(4), 551-575.

RILEY, J. G. (1979): "Informational Equilibrium,” Econometrica, 47(2), 331-359.

Rothschild, M., And J. Stiglitz (1976): "Equilibrium in Competitive Insurance Markets: An Essay on the Economics of Imperfect Information," Quarterly Journal of Economics, $90(4), 629-649$.

Spence, M. (1973): “Job Market Signalling," Quarterly Journal of Economics, 87(3), 355374.

WiLson, C. (1977): “A Model of Insurance Markets with Incomplete Information," Journal of Economic Theory, 16, 167-207. 